\title{
Molecular cloning and characterization of a bifunctional xylanolytic enzyme from Neocallimastix patriciarum
}

\author{
Cheng-Kang Pai $\cdot$ Zong-Yuan Wu $\cdot$ Ming-Ju Chen • \\ Yi-Fang Zeng $\cdot$ Jr-Wei Chen $\cdot$ Chung-Hang Duan • \\ Ming-Liang Li • Je-Ruei Liu
}

Received: 2 June 2009 /Revised: 29 July 2009 / Accepted: 29 July 2009 / Published online: 19 August 2009

(C) Springer-Verlag 2009

\begin{abstract}
A cDNA encoding a bifunctional acetylxylan esterase/xylanase, XynS20E, was cloned from the ruminal fungus Neocallimastix patriciarum. A putative conserved domain of carbohydrate esterase family 1 was observed at the N-terminus and a putative conserved domain of glycosyl hydrolase family 11 was detected at the C-terminus of XynS20E. To examine the enzyme activities, XynS20E was expressed in Escherichia coli as a recombinant $\mathrm{His}_{6}$ fusion protein and purified by immobilized metal ion-affinity chromatography. Response surface modeling combined with central composite design and regression analysis was then applied to determine the optimal temperature and $\mathrm{pH}$ conditions of the recombinant XynS20E. The optimal conditions for the highest xylanase activity of the recombinant XynS20E were observed at a temperature of $49^{\circ} \mathrm{C}$ and a $\mathrm{pH}$ of 5.8, while those for the highest carbohydrate esterase activity were observed at a temperature of $58^{\circ} \mathrm{C}$ and a $\mathrm{pH}$ of
\end{abstract}

Electronic supplementary material The online version of this article (doi:10.1007/s00253-009-2175-5) contains supplementary material, which is available to authorized users.

C.-K. Pai $\cdot$ M.-L. Li

Department of Life Science, National Taiwan Normal University, Taipei, Taiwan

Z.-Y. Wu • M.-J. Chen · Y.-F. Zeng • J.-W. Chen • J.-R. Liu ( $\varangle)$ Department of Animal Science and Technology, Institute of Biotechnology, National Taiwan University, 4F., No. 81, Chang-Xing Street,

Taipei, Taiwan

e-mail: jrliu@ntu.edu.tw

C.-H. Duan

Biotechnology Development Center,

Ye Cherng Industrial Products Co., LTD,

Yang Mei, Taiwan
8.2. Under the optimal conditions for the enzyme activity, the xylanase and acetylxylan esterase specific activities of the recombinant XynS20E toward birchwood xylan were 128.7 and $873.1 \mathrm{U} \mathrm{mg}^{-1}$, respectively. To our knowledge, this is the first report of a bifunctional xylanolytic enzyme with acetylxylan esterase and xylanase activities from rumen fungus.

Keywords Rumen $\cdot$ Neocallimastix patriciarum . Xylanase $\cdot$ Acetylxylan esterase

\section{Introduction}

Xylan constitutes the major component of hemicellulose and is the second most abundant renewable resource with a high potential for degradation to useful end products (Collins et al. 2005). Xylan is a heteropolysaccharide containing substituent groups of acetyl, 4- $O$-methyl-D-glucuronosyl, and $\alpha$-arabinofuranosyl residues linked to the backbone of $\beta-1,4$-xylopyranosyl units and has binding properties mediated by covalent and noncovalent interactions with cellulose, lignin, and other polymers (Subramaniyan and Prema 2002). As xylan varies in structure between different plant species, complete hydrolysis requires a large variety of cooperatively acting enzymes such as xylanases, xylosidases, arabinofuranosidases, glucuronidases, acetylxylan esterases, ferulic acid esterases, and $p$-coumaric acid esterases (Subramaniyan and Prema 2002; Collins et al. 2005). Of these xylanolytic enzymes, xylanase is of particular significance because it can catalyze the random hydrolysis of $\beta-1,4-$ xylosidic linkages in xylan to produce xylooligosaccharides, which are further degraded by other accessory enzymes. Among the accessory enzymes, acetylxylan esterase (EC 3.1.1.72) hydrolyzes specifically the ester linkages of the 
acetyl groups in position 2 and/or 3 of the xylose moieties of natural xylan and plays a rule in enhancing the accessibility of xylanase to the xylan backbone and subsequent hydrolysis of xylan (Dupont et al. 1996).

Xylanases are used in a range of industrial processes, such as biobleaching in the paper and pulp industry, bioconversion of lignocellulosic material and agro-wastes to fermentative products, clarification of juices, and improvement of the digestibility of animal feed stock (Subramaniyan and Prema 2002). As plant cell walls are structurally complex, a cocktail consisting of bi- and multifunctional xylanases and xylan debranching enzymes is the most desired combination for the efficient utilization of these complex materials. Some enzymes have evolved to possess bifunctional activity (Khandeparker and Numan 2008). Several genes encoding for bifunctional acetylxylan esterase/xylanase enzyme have been isolated from bacteria, including Clostridium cellulovorans (Kosugi et al. 2002), C. thermocellum (Blum et al. 2000), Cytophaga hutchinsonii (Xie et al. 2007), Pseudobutyrivibrio xylanivorans (Cepeljnik et al. 2006), and Ruminococcus flavefaciens (Aurilia et al. 2000). Studies on the structure of these bifunctional acetylxylan esterase/xylanase enzymes have revealed that they usually contain two or multiple different catalytic domains connected by linker sequences or noncatalytic sequences, some of which constitute cellulose binding domains (Fanutti et al. 1995).

Rumen fungi are able to degrade the most resistant plant cell-wall polymers (Selinger et al. 1996); thus, the rumen fungal population represents a rich and underutilized source of fibrolytic enzymes with tremendous potential for industrial and agricultural applications. Many xylanase genes have been cloned from a number of rumen fungal species, including Neocallimastix spp., Orpinomyces spp., and Piromyces spp. (Huang et al. 2005; Liu et al. 2008); however, only a few carbohydrate esterase genes have been cloned from rumen fungi (Blum et al. 1999; Dalrymple et al. 1997; Fillingham et al. 1999). To the best of our knowledge, a bifunctional acetylxylan esterase/ xylanase enzyme gene has never been cloned from rumen fungi.

In this study, we report the cloning and heterologous expression of cDNA encoding a bifunctional acetylxylan esterase/xylanase XynS20E from rumen fungus $N$. patriciarum S20. Response surface modeling (RSM) combined with central composite design (CCD) and regression analysis was then employed for the planned statistical optimization of the xylanase and acetylxylan esterase activities of the recombinant XynS20E. Substrate specificity and kinetic parameters, as well as the synergistic effect between the glycosyl hydrolase $(\mathrm{GH})$ domain and the carbohydrate esterase (CE) domain of the recombinant XynS20E were also studied.

\section{Materials and methods}

Cloning cDNA encoding the xylanase XynS20E

In a previous study, the xylanase-producing $N$. patriciarum S20 strain was isolated from rumen fluid of Taiwanese water buffalo (Bubalus bubalis) and the cDNA library of $N$. patriciarum S20 was constructed (Liu et al. 2008). In this study, the recombinant phages from the $N$. patriciarum S20 cDNA library were used to transfect Escherichia coli XL1Blue cells (BD Bioscience, Palo Alto, CA) and screened again for xylanase activity as described by Liu et al. (2008). The presence of a yellow halo was indicative of xylanase activity of the phages, which were then converted into plasmid form by Cre-recombinase-mediated excision. The resultant plasmids (pTriplEx2-S20E) were purified and the sequence of $x y n S 20 E$ insertion was determined by automatic sequencing (Mission Biotech Inc. Taipei). SignalP 3.0 (http://www.cbs.dtu.dk/services/SignalP-3.0/) was used to identify signal sequence cleavage sites. Sequences were aligned in the BioEdit Sequence Alignment Editor program (Hall 1999).

\section{Subcloning of $x y n S 20 E$}

To avoid confusion, the complete product of $x y n S 20 E$ is called XynS20E, the product of the CE domain is called XynS20E-CE1, and XynS20E-GH11 refers to the product of the glycosyl hydrolase (GH) domain. The cDNA sequences encoding XynS20E were amplified by PCR from pTriplEx2-S20E using the oligonucleotide forward primer: 5' CATATGAGAAACCTTGACAAACGTCAATG 3' and reverse primer: 5' CTCGAGATTTTTAACGT AAACCTTGGCG 3' (the underlined sequences in the primers are additional sequences that represent the restriction sites for NdeI and XhoI, respectively), while the cDNA sequences encoding XynS20E-CE1 were amplified by PCR from pTriplEx2-S20E using the same forward primer as in the amplification of XynS20E, and the reverse primer was replaced by the primer: 5' CTCGAGAGAAACTGGACC ATCTAC 3' (the underlined sequences in the primer are additional sequences that represent the restriction site for XhoI). The cDNA sequences encoding XynS20E-GH11 were amplified by PCR from pTriplEx2-S20E using the forward primer: 5' CATATGGAAAGTGTAACAGTTACT AGTAAC 3' (the underlined sequences in the primer are additional sequences that represent the restriction site for $\mathrm{NdeI}$ ) and the same reverse primer as in the amplification of XynS20E. The PCR fragments encoding XynS20E, XynS20E-CE1, and XynS20E-GH11 were digested with $N d e \mathrm{I}$ and $\mathrm{XhoI}$, and ligated with $\mathrm{NdeI}$-XhoI digested pET29a (Novagen, Madison, WI) to generate pET-xynS20E, pET-xynS20E-CE1, and pET-xynS20E-GH11, respectively. 
The products were then sequenced to ensure that no errors had been introduced by PCR. The resultant plasmids were used to transform E. coli BL21 (DE3; Novagen) by standard techniques (Sambrook and Russell 2001). Transformants were selected on LB agar plates containing kanamycin $\left(30 \mu \mathrm{g} \mathrm{ml}^{-1}\right.$; Sigma).

Purification of the recombinant XynS20E, XynS20E-CE1, and XynS20E-GH11

E. coli BL21 transformant cells were cultured in LB broth, and cell growth was then measured turbidimetrically at $600 \mathrm{~nm}$ (OD600). To produce the recombinant protein, the overnight culture was prepared and subsequently seeded at a 1:100 dilution into $5 \mathrm{ml}$ of fresh LB broth. The cell cultures were maintained at $37^{\circ} \mathrm{C}$ and induced with $100 \mu \mathrm{M}$ of IPTG (Sigma) for protein production upon reaching an OD600 of 0.5. After $4 \mathrm{~h}$ of induction, the cells were harvested by centrifugation at $5000 \times \mathrm{g}$ for $20 \mathrm{~min}$ at $4^{\circ} \mathrm{C}$.

The cell pellet was resuspended in $1 \mathrm{ml}$ of $0.1 \mathrm{M}$ sodium phosphate buffer ( $\mathrm{pH} 7.4$ ), sonicated for $10 \mathrm{~min}$ with an ultrasonicator (Model XL, Misonix, Farmingdale, NY), and fractioned into supernatant and pellet parts by subsequent centrifugation. The recombinant proteins were present mainly in the pellet and so were treated with $8 \mathrm{M}$ urea to induce protein unfolding. The proteins were then purified by immobilized metal ion-affinity chromatography using prepacked HisTrap Ni-Sepharose columns (GE Healthcare, New Jersey, USA). Finally, the soluble recombinant proteins were obtained by on-column refolding method using HiTrap desalting columns (GE Healthcare). The purified recombinant proteins were analyzed by sodium dodecyl sulfate polyacrylamide gel electrophoresis (SDS-PAGE) and then the enzyme activities were determined. Total protein concentration was measured using the Bradford protein assay (Bio-Rad Laboratories, Inc., Hercules, CA) against a standard curve of bovine serum albumin (Sigma).

Gel electrophoresis and zymogram analysis

SDS-PAGE analysis was performed according to the method described by Laemmli (1970). The zymogram technique was done according to the method described by Liu et al. (2008).

Optimum $\mathrm{pH}$ and temperature of enzyme activity of the recombinant XynS20E

RSM, its main effects and the interaction between the different factors at each level, was simultaneously investigated. CCD with two variables at five levels and five replicates at the central point, for a total of 13 experiments were conducted. According to our preliminary experimental results, $\mathrm{pH}$ and temperature were identified as the major factors affecting the enzyme activity of the recombinant $\mathrm{XynS20E}$ and were chosen as factors in the experimental design. In the statistical model, $Y_{1}$ denotes units of xylanase activity and the scaled values were defined as follows: $X_{1}=$ $(\mathrm{pH}-6) ; X_{2}=(T-50) / 10 ; Y_{2}$ denotes units of acetylxylan esterase activity and the scaled values were defined as follows: $X_{1}=(\mathrm{pH}-8) ; X_{2}=(T-60) / 10$. The experimental index number, scaled and real values are shown in Table 1 . The experimental design, data analysis and regression model building were performed using Design Expert software (version 7.13, Stat-Ease Inc., Minneapolis, MN). The responses, as linear, quadratic and cubic functions of the variables, were tested for adequacy and fitness using analysis of variance. Model analysis and the lack-of-fit test were used for selection of adequacy models. A model with $P$ values $(P>F)$ less than 0.05 was regarded as significant. The highest-order significant polynomial was selected. The lack-of-fit test was used to compare the residual and pure errors at replicated design points. The response predictor was discarded where lack-of-fit was significant, as indicated by a low probability value $(P>F)$. The model with no significant lack-of-fit was selected. Predicted residual sum of the squares (PRESS) was used as a measure of the fit of the model to the points in the design. The smaller the PRESS statistic is, the better the model fits the data points (Segurola et al. 1999).

After the optimal conditions for enzyme activity had been predicted, a series of experiments were conducted in triplicate and repeated three times in order to check the reliability of the predicted values and experimental data. The results were analyzed using Student's $t$ test available from the Statistical Analysis System software (SAS; version 8.1; Statistical Analysis System Institute, Cary, NC).

Enzyme activity assays

To determine the optimum $\mathrm{pH}$ and temperature of XynS20E activity, $5 \mu \mathrm{g}$ of the purified recombinant XynS20E was incubated with $0.5 \%(w / v)$ birchwood xylan (Sigma) in $100 \mathrm{mM}$ sodium citrate buffer (pH 4 to 5), sodium phosphate buffer (pH 6 to 8), or glycine sodium hydroxide buffer ( $\mathrm{pH} 9$ to 10) in a final reaction volume of $300 \mu \mathrm{l}$. After incubation for $20 \mathrm{~min}$ at the respective reaction temperature, xylanase activity was determined by measuring the amounts of reducing sugars released from the substrates using the dinitrosalicyclic acid (DNS) reagent method as described by Konig et al. (2002); acetylxylan esterase activity was determined by measuring the amount of acetic acid released from the substrates by using highperformance liquid chromatography (HPLC) as described by Blum et al. (1999). One unit of enzyme activity was defined as that releasing $1 \mu \mathrm{mol}$ of product/min from the 
Table 1 Variables used in the CCD, showing the treatment combinations and the mean experimental responses

\begin{tabular}{|c|c|c|c|c|c|c|c|c|}
\hline \multirow[t]{3}{*}{ Treatment } & \multirow{2}{*}{\multicolumn{2}{|c|}{ Coded setting levels $\left(X_{1}=\mathrm{pH} ; X_{2}=\mathrm{T}\right)$}} & \multicolumn{4}{|c|}{ Actual levels $\left(X_{1}=\mathrm{pH} ; X_{2}=\mathrm{T}\right)$} & \multicolumn{2}{|c|}{ Enzyme activity ${ }^{\mathrm{a}}$ (U/mg of total protein) } \\
\hline & & & \multicolumn{2}{|c|}{ Xylanase } & \multicolumn{2}{|c|}{ Acetylxylan esterase } & \multirow[t]{2}{*}{ Xylanase $^{\mathrm{b}}$} & \multirow[t]{2}{*}{ Acetylxylan esterase $^{c}$} \\
\hline & $X_{1}$ & $X_{2}$ & $X_{1}$ & $X_{2}$ & $X_{1}$ & $X_{2}$ & & \\
\hline 1 & -1 & -1 & 5.0 & 40 & 7.0 & 50 & 103.98 & 269.63 \\
\hline 2 & 0 & -1.41 & 6.0 & 36 & 8.0 & 45.86 & 80.11 & 351.54 \\
\hline 3 & -1.41 & 0 & 4.59 & 50 & 6.59 & 60 & 93.11 & 138.80 \\
\hline 4 & 0 & 0 & 6.0 & 50 & 8.0 & 60 & 107.29 & 796.91 \\
\hline 5 & 1 & 1 & 7.0 & 60 & 9.0 & 70 & 81.3 & 180.08 \\
\hline 6 & 0 & 0 & 6.0 & 50 & 8.0 & 60 & 124.23 & 801.27 \\
\hline 7 & 0 & 0 & 6.0 & 50 & 8.0 & 60 & 112.02 & 938.31 \\
\hline 8 & 1.41 & 0 & 7.41 & 50 & 9.41 & 60 & 81.3 & 380.75 \\
\hline 9 & 0 & 0 & 6.0 & 50 & 8.0 & 60 & 109.65 & 833.70 \\
\hline 10 & 1 & -1 & 7.0 & 40 & 9.0 & 50 & 95.47 & 586.77 \\
\hline 11 & 0 & 1.41 & 6.0 & 64 & 8.0 & 74.14 & 80.11 & 395.17 \\
\hline 12 & 0 & 0 & 6.0 & 50 & 8.0 & 60 & 121.47 & 516.91 \\
\hline 13 & -1 & 1 & 5.0 & 60 & 7.0 & 70 & 93.11 & 0 \\
\hline
\end{tabular}

${ }^{a}$ Results represent the mean of three experiments

${ }^{\mathrm{b}}$ Xylanase activity was determined by measuring the release of reducing sugars from birchwood xylan ( $\left.1 \% \mathrm{w} / \mathrm{v}\right)$ by DNS method

${ }^{\mathrm{c}}$ Acetylxylan esterase activity was determined by measuring the release of acetic acid from birchwood xylan ( $1 \%$ w/v) by HPLC method

substrate under the assay conditions. Specific activity was expressed as Units per milligram protein.

\section{Substrate specificity of the recombinant XynS20E}

In the assay to determine the substrate specificity of XynS20E, all reactions were conducted at the optimum $\mathrm{pH}$ and temperature of the recombinant XynS20E for xylanase activity or acetylxylan esterase activity. The recombinant XynS20E activities toward acetylxylan, beechwood xylan (Sigma), birchwood xylan (Sigma), or oat-spelt xylan (Sigma) were determined by measuring the amount of reducing sugars released from the substrates using the DNS reagent method as described above. Acetylxylan was prepared by the acetylation of oat-spelt xylan as described by Johnson et al. (1988). The amount of acetic acid produced by the action of the recombinant XynS20E on acetylxylan, beechwood xylan, birchwood xylan, oat-spelt xylan, or $\beta$-D-xylose tetraacetate (Sigma) was measured by HPLC as described above. Hydrolytic activity toward 4-methylumbelliferyl acetate (Sigma) was determined spectrophotometrically by measuring the release of 4-methylumbelliferone from 4methylumbelliferyl acetate at $354 \mathrm{~nm}$ during the initial 2 min period of the assay (Shao and Wiegel 1995). Activity towards $p$-nitrophenyl acetate (Sigma), $p$-nitrophenol laurate (Sigma), $p$-nitrophenol myristate (Sigma), $p$-nitrophenol palmitate (Sigma), and $\alpha$-naphthyl acetate (Sigma) was assayed as described previously (Blum et al. 1999).
Kinetic parameters of the recombinant XynS20E

To determine the kinetic parameters of XynS20E, birchwood xylan at concentration ranging from 1 to $6 \mathrm{mg} \mathrm{ml}^{-1}$ (for xylanase activity assay) or 10 to $60 \mathrm{mg} \mathrm{ml}^{-1}$ (for acetylxylan esterase activity assay) was incubated with $5 \mu \mathrm{g}$ of the recombinant XynS20E in a final reaction volume of $300 \mu$. Reactions were conducted at the optimal conditions for the highest xylanase activity or the highest carbohydrate esterase activity of recombinant XynS20E for $10 \mathrm{~min}$. A typical Lineweaver-Burk plot was obtained when $1[\nu]^{-1}$ was plotted against $1[S]^{-1}$ (Lineweaver and Burk 1934). Kinetic parameters $\left(K_{\mathrm{m}}\right.$ and $\left.V_{\max }\right)$ were estimated by linear regression from the Lineweaver-Burk plot.

Nucleotide sequence accession number

The nucleotide sequence of $x y n S 20 E$ has been submitted to the GenBank databases under accession number FJ529209.

\section{Results}

Cloning cDNA encoding the xylanase XynS20E

Three recombinant xylanase-producing phages were isolated from the cDNA library of $N$. patriciarum S20 by Congo red 
plate assay. The restriction map indicated that these xylanase-positive recombinants contained cDNA sequences derived from the same mRNAs. The cDNA sequence, designated as $x y n S 20 E$, contained a complete open reading frame of 2,016 bp with 5' and 3' untranslated regions of 162 and 243 bp, respectively (Fig. 1).

Amino acid sequences and domains of XynS20E

Translation of the open reading frame of xynS20E revealed a protein of 671 amino acids with a predicted molecular weight of $72.4 \mathrm{kDa}$. Database searches of the deduced amino acid sequence were performed using the National Center for Biotechnology Information's Basic Local Alignment Search Tool (BLAST). The deduced amino acid sequence matched several xylanases. According to the sequence-based $\mathrm{GH}$ classification, a putative conserved domain of GH family 11 was detected at the C-terminus of XynS20E (position 468-659; Fig. 1). The amino acid sequence alignment of the GH catalytic domain of XynS20E and other GH family 11 xylanases available from the GenBank showed that the highest identity is with xylanase from $N$. frontalis $(99.4 \%$, accession no. CAA58720), followed by xylanases from Piromyces sp. (90.5\%, accession no. CAA62969), Piromyces communis (87.7\%, accession no. AAG18439), N. patriciarum $(83.2 \%$, accession no. AAF14365), and Fibrobacter succinogenes (56.6\%, accession no. AAA21848).

According to the sequence-based CE classification, a putative conserved domain of CE family 1 was detected at the N-terminus of XynS20E (position 54-279; Fig. 1). The amino acid sequence alignment of $\mathrm{CE}$ catalytic domain of XynS20E and other CE family 1 esterases available from the GenBank showed that the highest identity is with carbohydrate esterase CHU_2040 from $C$. hutchinsonii (45.7\%, accession no. YP 678645), followed by carboxylesterase from Shewanella woodyi $(29.5 \%$, accession no. YP_001762318), lipoprotein from Myxococcus xanthus (25.6\%, accession no. YP_634438), Poly (3-hydroxybutyrate) depolymerase from Mycobacterium avium subsp. paratuberculosis (25.6\%, accession no. NP_963222), and carbohydrate esterase CHU_2408 from C. hutchinsonii (24.6\%, accession no. YP 679006).

Between the N-terminal CE family 1 catalytic domain and the C-terminal $\mathrm{GH}$ family 11 catalytic domain of XynS20E, two putative conserved dockerin domains were found (position 335-373 and 383-421; Fig. 1). The amino acid sequence alignment of this double dockerin showed that the highest sequence identity of XynS20E was $62 \%$ with that of XynA from Orpinomyces sp. PC-2 (accession no. AAD04194), followed by $60 \%$ with that of XynA from Piromyces sp. (accession no. Q12667), 58\% with that of XynWF1 from $P$. communis (accession no. ABY52795), and $58 \%$ with that of Xyn3 from $N$. frontalis (accession no. CAA57717).

Heterologous expression of $x y n S 20 E$ and purification of the recombinant XynS20E

PCRs were set up to subclone the DNA fragments of $x y n S 20 E$ into the pET-29a expression vector. XynS20E was expressed in $E$. coli as a recombinant $\mathrm{His}_{6}$ fusion protein. After induction with IPTG at $37^{\circ} \mathrm{C}$, the induced and noninduced recombinant bacteria were analyzed by SDSPAGE. A band of about $72 \mathrm{kDa}$ corresponding to the

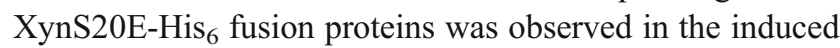
recombinant bacteria (Fig. 2A, lane 2). After centrifugation, the expressed recombinant proteins were predominately found in the insoluble fraction of cell lysate (Fig. 2A, lane 4). After treatment with $8 \mathrm{M}$ urea, the recombinant proteins dissolved (Fig. 2A, lane 5). The purified XynS20E-His 6 fusion proteins were obtained after purification by affinity chromatography and the desalting column (Fig. 2A, lane 6 and 7). The xylanase activity of purified recombinant XynS20E was further confirmed by zymographic analysis of xylan-SDS-PAGE. The recombinant XynS20E revealed a xylanase activity band of about $72 \mathrm{kDa}$ (Fig. 2B). The yield of the purified recombinant XynS20E was 43.16 $5.71 \mu \mathrm{g}$, starting from $130 \mathrm{mg}$ (wet weight) of $E$. coli cells.

Optimization of enzyme activity of the recombinant XynS20E

The classical method of 'one-variable-at-a-time' bioprocess design may be effective in some situations, but fails to consider the combined effects of all factors involved (Heck et al. 2006). RSM is an empirical modeling technique used to evaluate the relationship between a set of controllable experimental factors and observed results. The CCD experimental design, which minimizes the number of experimental runs, was used to determine the effects of independent variables on the dependent variables. According to our preliminary experimental results, temperature and $\mathrm{pH}$ were identified as the major factors affecting the xylanase activity of the recombinant XynS20E. The process variables used in the experimental design and results for enzyme activities are shown in Table 1. Treatments 4, 6, 7, 9, and 12 (central points) showed the highest levels of xylanase activity $(107.29,124.23,112.02,109.65$, and $121.47 \mathrm{U} \mathrm{mg}^{-1}$ of total protein, respectively) and acetylxylan esterase activity $(796.91,801.27,938.31,833.70$, and $516.91 \mathrm{U} \mathrm{mg}^{-1}$ of total protein, respectively).

Table 2 compares the validities of the linear, quadratic and cubic models for the responses according to their $F$ values. The quadratic model for xylanase activity as well as for acetylxylan esterase activity of the recombinant 
Fig. 1 Nucleotide sequence of the cDNA encoding the acetylxylan esterase/xylanase enzyme XynS20E, its flanking regions and the deduced amino acid sequence. The deduced amino acid sequence of the $x y n S 20 E$ product is indicated in boldface. The translational stop codon is indicated by an asterisk $(*)$. The signal peptide sequence is underlined. The putative region of carbohydrate esterase family 1 is shaded. The putative region of glycosyl hydrolase family 11 is double-underlined. The putative regions of dockerin domains are boxed
GGTTCTAAAATAATTAATAATTATTAATATTATTTTCTCTGGAAGAAAATAGTTTATATTGTAAAAAAGAAA aTAAATTGAAATTTTTAAAAAaATAAAAATTAAAATAATAAaGTAAATAGTTTAAAAATTTTAATTATTAATATTTTAAAAAAATCAA ATGAGATTAGGTGTTGCTTTAAGTACTATTGCAGTTTTATTAACTGCTACATCTGCTAGAAACCTTGACAAACGTCAATGGGGATGGCCT

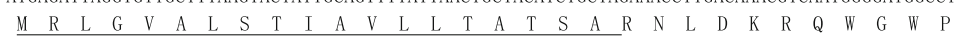

AATTTCGGTGGTGGTAATGGTGGTAATGGTGGTAATGGTGGTAAAACCATCAATGACTACAAAAGAGAACAAGGAGCTGGTAGAGATATT

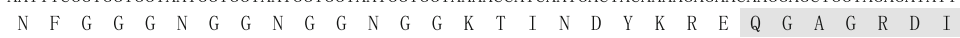
CATGTTTATGCCCCATCAAATCTTGCTCCAAATAGTCCATTACTTCTTTCTTTACACGGTATGGACCAAGATCCAAATTATCAACAATCA

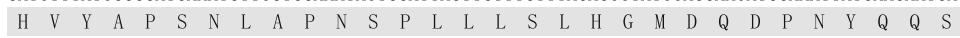
AACACTCACTGGGAAACTTTAGCTGATTCTGAAGGTTTTGTTGTTGTTTATCCAAGAGGTGGAACTGGTATGAGTACTTGGGATATTCAA $\begin{array}{llllllllllllllllllllllllllllllll}N & T & H & W & E & T & L & A & D & S & E & G & F & V & V & V & Y & P & R & G & G & T & G & M & S & T & W & D & I & Q\end{array}$ GGTACTAAGGATACTCAATGGGTCAGTCAAATTATTGATCAAATGAAAAAGGAATACAATATTGATACCAAGCGTGTATACCTTTCTGGT $\begin{array}{llllllllllllllllllllllllllllllll}G & T & K & D & T & Q & W & V & S & Q & I & I & D & Q & M & K & K & E & Y & N & I & D & T & K & R & V & Y & L & S & G\end{array}$

TTCTCTATGGgTGGTATGTTTACTTACCATGCTATGAGCCAAATTGCTAATAAGATTGCTGCTTTTGCTCCATGTTCAGGTCCAAATGTA $\begin{array}{llllllllllllllllllllllllllllll}F & S & M & G & G & M & F & T & Y & H & A & M & S & Q & I & A & N & K & I & A & A & F & A & P & C & S & G & P & N & V\end{array}$

TTTGGTGCCTCAAAAGCCCAACGTCCAGTTCCAATTTTCCACGTTCATGGAACTAATGATGATGTTTTGAATTATCAACAAGTTGAAGGT \begin{tabular}{llllllllllllllllllllllllllllllll}
\hline & $G$ & $A$ & $S$ & $K$ & $A$ & $Q$ & $R$ & $P$ & $V$ & $P$ & $I$ & $F$ & $H$ & $V$ & $H$ & $G$ & $T$ & $N$ & $D$ & $D$ & $V$ & $L$ & $N$ & $Y$ & $Q$ & $Q$ & $V$ & $E$ & $G$
\end{tabular}

TTCCTTAAGAATTACCGTGATCAATTCCATTGTCCTTCACAAGCTGATACCAAGACAAACTATCCGAACAGAGAAAATCCAAATGCTACT \begin{tabular}{llllllllllllllllllllllllllllllll}
\hline & $L$ & $L$ & $K$ & $N$ & $Y$ & $R$ & $D$ & $Q$ & $F$ & $H$ & $C$ & $P$ & $S$ & $Q$ & $A$ & $D$ & $T$ & $K$ & $T$ & $N$ & $Y$ & $P$ & $N$ & $R$ & $E$ & $N$ & $P$ & $N$ & $A$ & $T$
\end{tabular}

CTTTACACTTGGGGTCCATGTGATAAGGGAGTTTATATTAAGCACTTAAAGCTCCAAGGTCGTGGACACAGTCCATCTTCTGCTGATATT $\begin{array}{llllllllllllllllllllllllllllllll}L & \text { Y } & T & \text { W } & G & P & C & D & K & G & V & Y & I & K & H & L & K & L & Q & G & R & G & H & S & P & S & S & A & D & I\end{array}$

CAAGATATTTGgGATTTCGTTAGTCAATGGACTGTAGATGGTCCAGTTTCTGCCAGTGGAAACGgTGGTGGTAACACTACTCCAACTAAC $\begin{array}{lllllllllllllllllllllllllllllll}Q & D & I & W & D & F & V & S & Q & W & T & V & D & G & P & V & S & A & S & G & N & G & G & G & N & T & T & P & T & N\end{array}$

CCATCTACTGGTGGTAACGGAAACGGAAATGGTGGTGGTAACACTACTCCAACTAACCCATCTACTGGTGGTAACGGAAACGGAAATGGT $\begin{array}{llllllllllllllllllllllllllllll}P & S & T & G & G & N & G & N & G & N & G & G & G & N & T & T & P & T & N & P & S & T & G & G & N & G & N & G & N & G\end{array}$

GGTAGCACTGATAAATGTTCTTCTAATATTACTAAACAGGGATACAAATGTTGTGCTAGCAATTGTGAAGTAGTTTACACTGACAGTGAT \begin{tabular}{lllllllllllllllllllllllllllllll}
$G$ & $S$ & $T$ & $D$ & $K$ & $C$ & $S$ & $S$ & $N$ & $I$ & $T$ & $K$ & $Q$ & $G$ & $Y$ & $K$ & $C$ & $C$ & $A$ & $S$ & $N$ & $C$ & $E$ & $V$ & $V$ & $Y$ & $T$ & $D$ & $S$ & $D$ \\
\hline
\end{tabular}

GGTGATTGGGGTGTTGAAAACGACCAATGGTGTGGATGTGGAAACAGAGTTACTGTTGGTTCTGGTACTTGTTCAGCAAAGATTCTTCAA

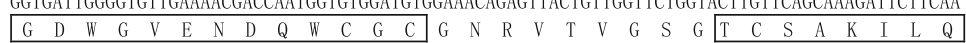
CAAGGATACAAATGTTGTCCATCTGGTTGTATTATTTACTACACTGATGAAGATGGTACTTGGGGTGTTAATGGTGAAGAATGGTGTGGT \begin{tabular}{|lllllllllllllllllllllllllllllll}
\hline$Q$ & $G$ & $Y$ & $K$ & $C$ & $C$ & $P$ & $S$ & $G$ & $C$ & I & I & Y & Y & T & D & E & D & $G$ & T & W & $G$ & V & N & $G$ & E & E & W & $C$ & $G$ \\
\hline
\end{tabular} TGTGGTTCCGGCAGTTCATCCACTGGTGGTGGTAACGATGCTCCATCAAGTGGTAGTGGATACCAAGGTGCTAATGGAACTAACTTCTGT \begin{tabular}{llllllllllllllllllllllllllllll}
\hline$C$ & $G$ & $S$ & $G$ & $S$ & $S$ & $S$ & $T$ & $G$ & $G$ & $G$ & $N$ & $D$ & $A$ & $P$ & $S$ & $S$ & $G$ & $S$ & $G$ & $Y$ & $Q$ & $G$ & $A$ & $N$ & $G$ & $T$ & $N$ & $F$ & $C$
\end{tabular} AACAACGCTAAGCACTCTGGTGAAAGTGTAACAGTTACTAGTAACAAGGTAGGTGATATCAACGGAATTGGATATGAATTATGGGCTGAT \begin{tabular}{llllllllllllllllllllllllllllll}
$N$ & $N$ & $A$ & $K$ & $H$ & $S$ & $G$ & $E$ & $S$ & $V$ & $T$ & $V$ & $T$ & $S$ & $N$ & $K$ & $V$ & $G$ & $D$ & $I$ & $N$ & $G$ & $I$ & $G$ & $Y$ & $E$ & $L$ & $W$ & $A$ & $D$ \\
\hline
\end{tabular} AGTGGTAACAATAGTGCAACTTTCTATGATGATGGTTCATTCTCTTGTTCATTCCAACGTGCTAAGGATTACTTATGTCGTTCCGGTCTT

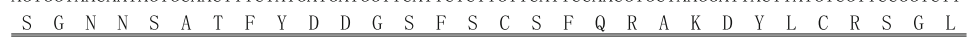
TCCTTTGATAGTACTAAGACTCACAAGCAAATTGGTCACATTTACGCTGAaTTCAAACTTGTTAAGCAAAACATTCAAAATGTAGATTAT \begin{tabular}{llllllllllllllllllllllllllllll}
$S$ & $F$ & $D$ & $S$ & $T$ & $K$ & $T$ & $H$ & $K$ & $Q$ & $I$ & $G$ & $H$ & $I$ & $Y$ & $A$ & $E$ & $F$ & $K$ & $L$ & $V$ & $K$ & $Q$ & $N$ & $I$ & $Q$ & $N$ & $V$ & $D$ & $Y$ \\
\hline \hline
\end{tabular} TCTTATGTTGGTATTTATGGTTGGACCAGAAATCCACTTGTTGAATTTTACGTTGTTGATAACTGGCTCAGTCAATGGCGTCCAGGAGAT \begin{tabular}{llllllllllllllllllllllllllllll}
$S$ & $Y$ & $V$ & $G$ & $I$ & $Y$ & $G$ & $W$ & $T$ & $R$ & $N$ & $P$ & $L$ & $V$ & $E$ & $F$ & $Y$ & $V$ & $V$ & $D$ & $N$ & $W$ & $L$ & $S$ & $Q$ & $W$ & $R$ & $P$ & $G$ & $D$ \\
\hline \hline
\end{tabular}

TGGGTTGGTAACAAGAAGCACGGTGATTTCACTATTGGTGGTGCTCAATACACTGTTTATGAAAATACTCGTTACGGTCCATCTATTGAC

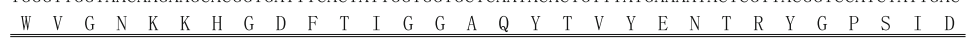

GGTGACACCAATTTCAAGCAATACTTCAGTATTCGTCAACAACCACGTGACTGTGGTACTATTGATATTACTGCTCATTTCGAACAATGG

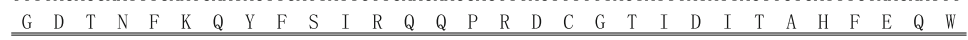

GAAAAGCTTGGTATGACTATGGGTAAGATGCACGAAGCTAAGGTTTTAGGTGAAGCCGGTAGTAACAATGGTGGTACATCTGGTACTGCT \begin{tabular}{lllllllllllllllllllllllllllllll}
\hline & $K$ & $L$ & $G$ & $M$ & $T$ & $M$ & $G$ & $K$ & $K$ & $H$ & $E$ & $A$ & $K$ & $V$ & $L$ & $G$ & $E$ & $A$ & $G$ & $S$ & $N$ & $N$ & $G$ & $G$ & $T$ & $S$ & $G$ & $T$ & $A$
\end{tabular}

GATTTCCCATTCGCCAAGGTTTACGTTAAAAATTAAATAGTTTCATAATAATGAAACCAATAAATACAAACTTTAGAAGTTTTAAAAATA D F P P F A

TATTAAAAAAAATTAAATGGGATTTTAAAATAATATAAAAATAAAGCAAAAAAAATATTGTAAAGAAACCTATCGTATAATTTTAAAAAC TTAGAAGTTATAAAATTATATTAAAAAAAAATTGGTAAATTAAATTAAATTTTAAATAACATAAAATAAACAAAAAAAAAAAAAAAAAAA AAAAAAAAA
72

162 252 30
XynS20E appeared to be the most accurate, with a statistically significant model analysis $(P<0.05)$ and insignificant lack-of-fit $(P>0.05)$. In addition, the goodness of fit of the quadratic model for xylanase or acetylxylan esterase activity was checked using the coefficient of determination $\left(R^{2}=0.8311\right.$ and 0.8353 , respectively), indicating that $83.11 \%$ of the total variation for xylanase activity or $83.53 \%$ of the total variation for acetylxylan 
A

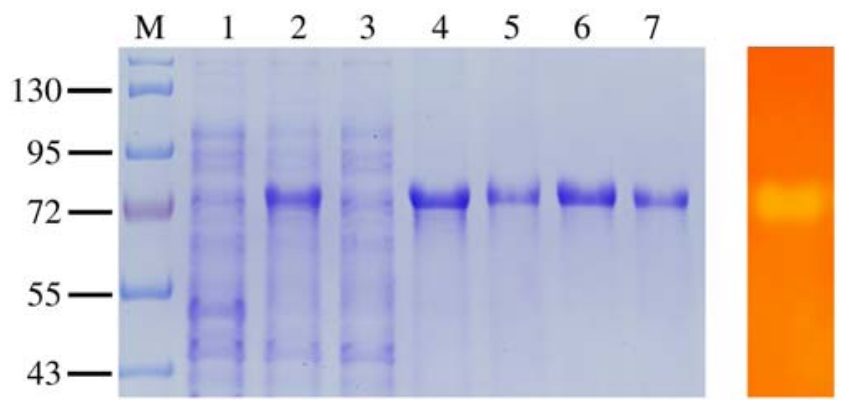

Fig. 2 SDS-PAGE and zymographic analysis of the recombinant XynS20E. (A) SDS-PAGE of expressed and purified protein encoded by xylanase gene. Lane $M$ molecular weight marker, lane 1 cell lysate of the recombinant $E$. coli before IPTG induction, lane 2 cell lysate of the recombinant $E$. coli after IPTG induction, lane 3 supernatant fraction of the cell lysate after centrifugation, lane 4 pellet fraction of the cell lysate after centrifugation, lane 5 soluble fraction after the pellet was treated with $8 \mathrm{M}$ urea, lane 6 urea-soluble XynS20E after HisTrap chromatography, lane 7 purified XynS20E after HiTrap chromatography $(2 \mu \mathrm{g}$ of protein in each lane). $(B)$ Zymogram of the purified XynS20E showing xylanase activity

esterase activity could be explained by the model. This confirms that the accuracy and general ability of the quadratic model was good, and analysis of the associated response trends was reasonable.
The quadratic model generated by the design is:

$$
\begin{aligned}
Y_{1}= & -651.198+136.437 \times \mathrm{pH}+15.141 \times T-11.412 \\
& \times \mathrm{pH}^{2}-0.150 \times T^{2}-0.083 \times \mathrm{pH} \times T \\
Y_{2}= & -26519.661+4681.454 \times \mathrm{pH}+279.402 \times T \\
& -273.184 \times \mathrm{pH}^{2}-2.164 \times T^{2}-3.427 \times \mathrm{pH} \times T
\end{aligned}
$$

where $Y_{1}$ is the predicted response for xylanase activity (units per milligram of total protein), $Y_{2}$ is the predicted response for acetylxylan esterase activity (units per milligram of total protein), and $\mathrm{pH}$, and $T$ are the actual values for $\mathrm{pH}$ and temperature (see Table 1).

The significance of the coefficients determined by the Student's $t$ test and the related $P$ values are presented in Table 3. The latter were used to check the significance of each coefficient, and to test the strength of the interaction between each independent variable (i.e., the smaller the $P$ value, the more significant the corresponding coefficient) (Akhnazarova and Kafarov 1982). In this study, the $P$ value of second-order $\mathrm{pH}$ and second-order $T$ of the quadratic model for xylanase and acetylxylan esterase activity were

\begin{tabular}{|c|c|c|c|c|c|c|c|c|}
\hline \multirow[t]{2}{*}{ Source of variation } & \multicolumn{2}{|l|}{ Xylanase } & \multicolumn{2}{|c|}{ Acetylxylan esterase } & \multicolumn{2}{|l|}{ Xylanase } & \multicolumn{2}{|c|}{ Acetylxylan esterase } \\
\hline & Sum of squares & $P>F$ & Sum of squares & $P>F$ & $R$-square & PRESS & $R$-square & PRESS \\
\hline
\end{tabular}
highly significant $(P<0.01)$. The high significance of the $\mathrm{pH}$ and $T$ second-order models indicates that they can act as

Table 2 Model analysis (a), lack-of-fit tests (b), $R$ square analysis (c) for measured responses

(a) Model analysis ${ }^{\mathrm{a}}$

$\begin{array}{lrlrl}\text { Mean } & 126,700.00 & & 2,947,000.00 & \\ \text { Linear } & 249.70 & 0.6415 & 135,300.00 & 0.5073 \\ \text { Quadratic } & 2,190.08 & 0.0027^{\mathrm{b}} & 750,400.00 & 0.0030^{\mathrm{b}} \\ \text { Cubic } & 80.01 & 0.6444 & 71,089.69 & 0.2732 \\ \text { Residual } & 416.35 & & 104,500.00 & \\ \text { Total } & 129,600.00 & & 4,013,000.00 & \\ \text { (b) Lack of fit } & & & & \\ \text { Linear } & 2,465.19 & 0.0371^{\mathrm{d}} & 832,800.00 & 0.0574 \\ \text { Quadratic } & 272.39 & 0.3183 & 77,689.54 & 0.4594 \\ \text { Cubic } & 192.37 & 0.1374 & 6,599.86 & 0.6309 \\ \text { Pure error } & 223.98 & & 626,200.00 & \end{array}$

(c) $R$ square analysis ${ }^{\mathrm{e}}$

Linear
Quadratic
Cubic

${ }^{a}$ Model analysis: select the highest-order polynomial where the additional terms are significant

${ }^{\mathrm{b}}$ Statistically significant at $99 \%$ of confidence level

${ }^{\mathrm{c}}$ Lack of fit: want the selected model to have insignificant lack of fit

${ }^{\mathrm{d}}$ Statistically significant at $95 \%$ of confidence level

${ }^{\mathrm{e}} R$ square analysis: focus on the model minimizing the "PRESS" 
Table 3 Coefficient estimates by the regression model

\begin{tabular}{|c|c|c|c|c|c|c|}
\hline \multirow[t]{2}{*}{ Factor } & \multicolumn{3}{|l|}{ Xylanase } & \multicolumn{3}{|l|}{ Acetylxylan esterase } \\
\hline & Coefficient estimate & Standard error & $P$ value & Coefficient estimate & Standard error & $P$ value \\
\hline Intercept & 114.93 & 3.77 & & 777.42 & 70.82 & \\
\hline $\mathrm{pH}$ & -4.63 & 2.98 & 0.1640 & 104.92 & 55.99 & 0.1031 \\
\hline$T$ & -3.13 & 2.98 & 0.3280 & -76.83 & 55.99 & 0.2124 \\
\hline $\mathrm{pH} \times \mathrm{pH}$ & -11.41 & 3.19 & $0.0090^{\mathrm{a}}$ & -273.18 & 60.04 & $0.0026^{\mathrm{a}}$ \\
\hline$T \times T$ & -14.93 & 3.19 & $0.0022^{\mathrm{a}}$ & -216.39 & 60.04 & $0.0087^{\mathrm{a}}$ \\
\hline $\mathrm{pH} \times T$ & -0.82 & 4.21 & 0.8502 & -34.26 & 79.18 & 0.6782 \\
\hline
\end{tabular}

${ }^{\text {a }}$ Statistically significant at $99 \%$ of confidence level

limiting factors, with even small variations substantially altering xylanase and acetylxylan esterase activity (Heck et al. 2006).

The RSMs for xylanase and acetylxylan esterase activity as a function of $\mathrm{pH}$ and temperature of the recombinant XynS20E are depicted in Fig. 3. The results indicate that the optimal conditions for the xylanase activity of the recombinant XynS20E occur at $49^{\circ} \mathrm{C}$ and $\mathrm{pH} 5.8$ (Fig. 3a) while the optimal conditions for the acetylxylan esterase activity of the recombinant XynS20E occur at $58^{\circ} \mathrm{C}$ and $\mathrm{pH}$ 8.2 (Fig. 3b). To confirm the applicability of the model, xylanase and acetylxylan esterase activities at the suggested optimum conditions were determined. In the respective optimum condition, the model predicted a xylanase activity of $115.55 \mathrm{U} \mathrm{mg}^{-1}$ (range from 111.82 to 119.28) and an acetylxylan esterase activity of $795.57 \mathrm{U} \mathrm{mg}^{-1}$ (range from 725.65 to 865.49 ) at a confidence level of $95 \%$. The experimental xylanse activity of $128.7 \pm 32.9 \mathrm{U} \mathrm{mg}^{-1}$ and acetylxylan esterase activity of $873.1 \pm 18.9 \mathrm{U} \mathrm{mg}^{-1}$ confirmed the accuracy of the models.

\section{Substrate specificity and kinetic analysis}

XynS20E hydrolyzed xylan from acetylxylan, beechwood, birchwood, and oat-spelt, and released acetate from 4methylumbelliferyl acetate and $\beta$-D-xylose tetraacetate (Table 4). Under the optimal conditions for the enzyme activity, the xylanase specific activity of the purified recombinant XynS20E toward birchwood xylan was $128.7 \mathrm{U} \mathrm{mg}^{-1}$ with $K_{\mathrm{m}}$ of $1.48 \pm 0.42 \mathrm{mg} \mathrm{ml}^{-1}$ and a $V_{\max }$ of $153.27 \pm 25.02 \mu \mathrm{mol} \mathrm{min} \cdot \mathrm{mg}^{-1}$, while the acetylxylan esterase specific activity of the purified recombinant XynS20E toward birchwood xylan was $873.1 \mathrm{U} \mathrm{mg}^{-1}$ with $K_{\mathrm{m}}$ of $16.72 \pm 2.37 \mathrm{mg} \mathrm{ml}^{-1}$ and a $V_{\max }$ of $5.15 \pm$ $0.29 \mu \mathrm{mol} \mathrm{min} \cdot \mathrm{mg}^{-1}$. No activity was observed against carboxy methyl cellulose, $\beta$-glucan, $p$-nitrophenol acetate, $p$-nitrophenol laurate, $p$-nitrophenol myristate, $p$-nitrophenol palmitate, or $\alpha$-naphthol acetate.
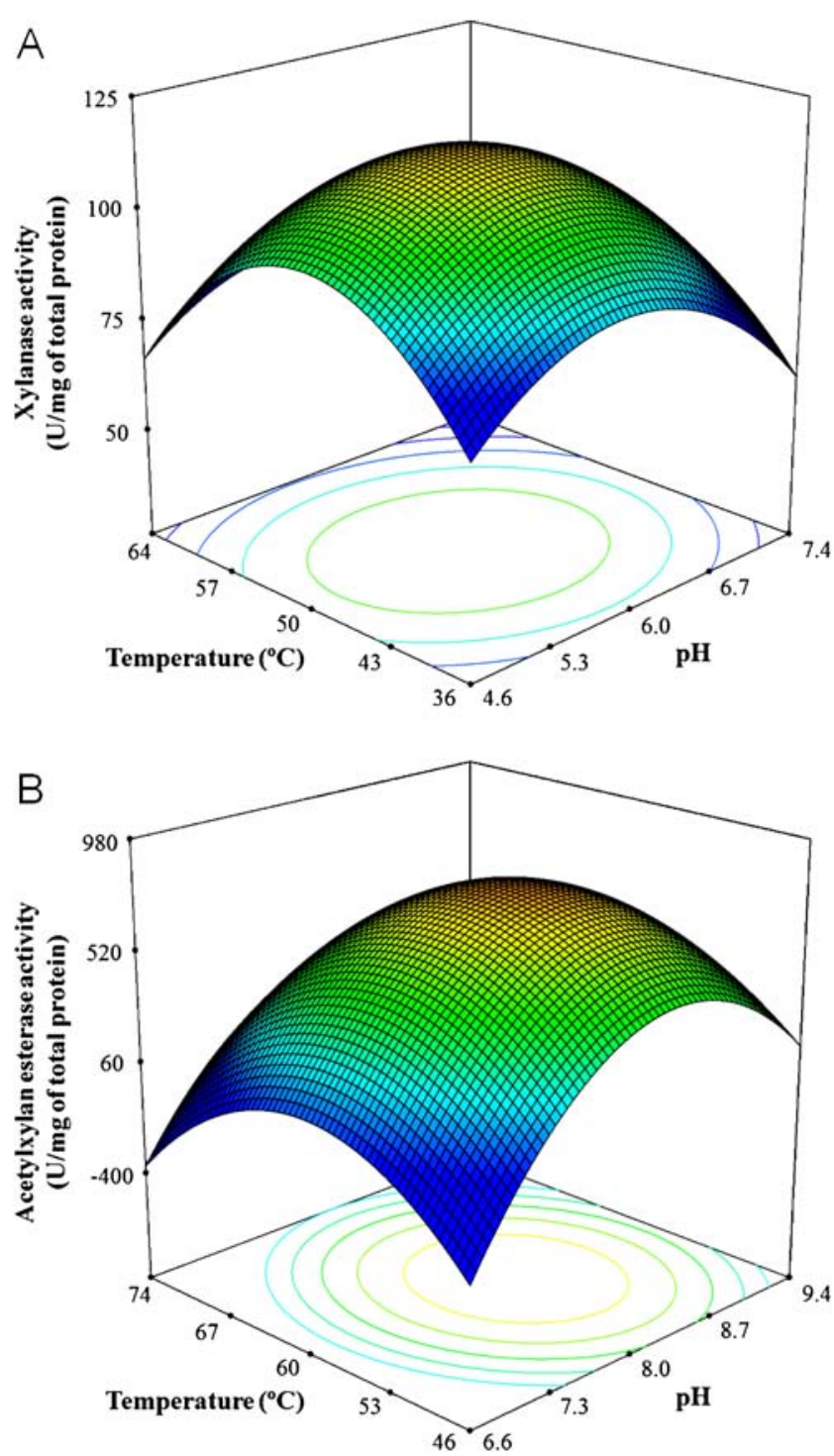

Fig. 3 Response surface plot of the effects of $\mathrm{pH}$ and temperature on xylanase activity $(A)$ and acetylxylan esterase activity $(B)$ of the recombinant XynS20E 
Table 4 Substrate specificity of the purified recombinant XynS20E

\begin{tabular}{lll}
\hline Substrate & \multicolumn{2}{l}{ Specific activity (Units per milligram of total protein) } \\
\cline { 2 - 3 } & Xylanase $^{\mathrm{b}}$ & Acetylxylan esterase $^{\mathrm{c}}$ \\
\hline Acetylxylan & $80.2 \pm 9.7$ & $2613.5 \pm 55.3$ \\
Beechwood xylan & $131.9 \pm 10.0$ & $273.7 \pm 48.3$ \\
Birchwood xylan & $128.7 \pm 32.9$ & $873.1 \pm 18.9$ \\
Oat-spelt xylan & $131.3 \pm 15.0$ & $557.9 \pm 81.2$ \\
4-methylumbelliferyl acetate & $\mathrm{ND}^{\mathrm{d}}$ & $580.3 \pm 62.5$ \\
$\beta$-D-xylose tetraacetate & $\mathrm{ND}$ & $1227.7 \pm 147.3$ \\
\hline
\end{tabular}

${ }^{\text {a }}$ Results represent the mean of three experiments

${ }^{\mathrm{b}}$ Xylanase activity was determined by measuring the release of reducing sugars from respective substrate $(1 \% w / v)$ at $49^{\circ} \mathrm{C}$, $\mathrm{pH} 5.8$ using the $\mathrm{DNS}$ reagent method

${ }^{\mathrm{c}}$ Acetylxylan esterase activity was determined by measuring the release of acetic acid from respective substrate $(1 \% \mathrm{w} / \mathrm{v})$ at $58^{\circ} \mathrm{C}$, $\mathrm{pH} 8.2 \mathrm{using}$ the HPLC method

${ }^{\mathrm{d}}$ Not detectable

The synergistic effect between the GH domain and CE domain of XynS20E in xylan degradation

The possibility of synergy between the acetylxylan esterase activity of the CE domain and the xylanase activity of the GH domain of XynS20E was studied with birchwood xylan as substrate. As a result, the xylanase activity of the recombinant XynS20E was more than twice that of XynS20E-GH11 for birchwood xylan, while the acetylxylan esterase activity of the recombinant XynS20E was about twice that of XynS20E-CE1 for birchwood xylan (Table 5). In addition, the recombinant XynS20E and XynS20E-CE1 could release acetic acid from birchwood

Table 5 Comparison of xylanase and acetylxylan esterase activities for each domain of XynS20E

\begin{tabular}{lll}
\hline Domain & \multicolumn{2}{l}{${\text { Relative activity rate }(\%)^{\mathrm{a}}}^{\mathrm{a}}$} \\
\cline { 2 - 3 } Xylanase $^{\mathrm{b}}$ & Acetylxylan esterase $^{\mathrm{c}}$ \\
\hline XynS20E & 100 & 100 \\
XynS20E-CE1 & $\mathrm{ND}^{\mathrm{d}}$ & 56 \\
XynS20E-GH11 & 49 & $\mathrm{ND}$
\end{tabular}

${ }^{a}$ The rate of enzyme activity are expressed relative to the amount of product liberated per micromole of protein when the recombinant XynS20E was incubated with birchwood xylan, which was set at $100 \%$

${ }^{\mathrm{b}}$ Xylanase activity was determined by measuring the release of reducing sugars from birchwood xylan $(1 \% \mathrm{w} / \mathrm{v})$ at $49^{\circ} \mathrm{C}$, $\mathrm{pH} 5.8$ using the DNS reagent method

${ }^{c}$ Acetylxylan esterase activity was determined by measuring the release of acetic acid from birchwood xylan $(1 \% \mathrm{w} / \mathrm{v})$ at $58^{\circ} \mathrm{C}, \mathrm{pH} 8.2$ using the HPLC method

${ }^{\mathrm{d}}$ Not detectable xylan while the recombinant XynS20E-GH11 did not show acetylxylan esterase activity (Table 5).

\section{Discussion}

Xylanases have potential application in many industries and have received tremendous attention. A thorough review of the relevant literature revealed that at least 30 different xylanase genes have been cloned from ruminal fungi, including genera of Neocallimastix, Orpinomyces, and Piromyces. According to the CAZy database (http://www. cazy.org/), xylanases are found in families $5,7,8,10,11$, 26 , and 43 , while all of the rumen fungal xylanases are found only in families 10 and 11 . Two of the conserved regions in GH family 11 xylanases, [PSA]-[LQ]-x-E-[YF]$\mathrm{Y}$-[LIVM](2)-[DE]-x-[FYWHN] and [LIVMF]-x(2)-E[AG]- [YWG]-[QRFGS]-[SG]-[STAN]-G-x-[SAF], are known as active site signatures 1 and 2, respectively (PROSITE database; http://www.expasy.org/prosite/). The glutamic acid residues centered in the active site signatures of GH family 11 xylanase have been identified as catalytic residues on the basis of three-dimensional models and mutational analysis (Wakarchuk et al. 1994). A notable exception is XynA from Piromyces sp., which is considered to belong to the GH family 11 though it lacks the conserved region of the active site signature 2 (Fanutti et al. 1995). Although XynS20E lacks the conserved region of the active site signature 2 , we suggest that it should be classified as members of GH family 11.

In contrast to xylanases, relatively little information on acetylxylan esterases is available. Biologically, acetylxylan esterases are involved in the removal of $O$-acetyl moieties from xylan and thus allow accessibility of the substrate to 
xylanase (Dupont et al. 1996). Among the 16 different families of carbohydrate esterase present in the CAZy database, acetylxylan esterases are found in families 1, 2, 3, $4,5,6,7$, and 12 . To date, only a few studies have focused on the rumen fungal carbohydrate esterases (Blum et al. 1999; Dalrymple et al. 1997; Fillingham et al. 1999). These carbohydrate esterases belong to families $1,2,3$, and 6 on the basis of the CAZy classification system, and most of them have been demonstrated to act synergistically with xylanase (Blum et al. 1999; Dalrymple et al. 1997; Fillingham et al. 1999). Nevertheless, none of the rumen fungal carbohydrate esterases possess the ability to hydrolyze xylan themselves. Some bacterial xylanolytic enzymes possess both xylanase and carbohydrate esterase activities in the same polypeptide chain (Aurilia et al. 2000; Blum et al. 2000; Cepeljnik et al. 2006; Kosugi et al. 2002; Xie et al. 2007); however, bifunctional acetylxylan esterase/ xylanase enzymes from rumen fungus have never been reported. Thus, XynS20E reported on herein is the first rumen fungal bifunctional acetylxylan esterase/xylanase to be discovered.

XynS20E resembles other reported acetylxylan esterases in exhibiting activity towards 4-methylumbelliferyl acetate (Ding et al. 2007; Ferreira et al. 1993; Halgasova et al. 1994). Interestingly, XynS20E showed no detectable activity on generic esterase substrates including nitrophenyl acetate in contrast with other esterases from the fungal family $1 \mathrm{CE}$ (Fillingham et al. 1999); this observation is similar to the acetylxylan esterase from Volvariella volvacea, which belongs to family CE1 and showed no activity towards nitrophenyl acetate (Ding et al. 2007). In addition, XynS20E showed high activity towards sugar-based substrates such as $\beta$-D-xylose tetraacetate and acetylxylan, thereby confirming that XynS20E is a true acetylxylan esterase.

Some rumen fungi may produce high-molecular-mass fibrolytic enzyme complexes similar to cellulosomes of anaerobic bacteria. It has been suggested that the fungal cellulosomes have scaffoldins that bind enzymatic subunits through interactions between cohesion domains of the scaffoldins and dockerins in the catalytic subunits (Fanutti et al. 1995). Three types of fungal dockerin sequences have been identified. Types 1 and 3 contain six cysteines, and type 2 contains four cysteines (Steenbakkers et al. 2001). Both of the dockerin domains of XynS20E were composed of 39 amino acids and contained six cysteines. Based on the number and position of the cysteine residues, both dockerin domains of XynS20E were classified as type I fungal dockerins. The fungal dockerins are generally duplicated in cellulosomal proteins (Steenbakkers et al. 2001). Comparison of the double-dockerin sequences showed that the length of the linker between the domains ranges from two to 11 amino acid residues (Raghothama et al. 2001). Nagy et al. (2007) demonstrated that tandem arrays of dockerin domains can bind more tightly and more extensively to the cellulosome than a single form of dockerin domain. Furthermore, if the linker connecting the two dockerin domains is short enough to keep the binding sites of the two domains on adjacent surfaces, the double-dockerin construct can bind more tightly to cellulosomes than a single domain and with greater coverage (Nagy et al. 2007). XynS20E contains a doubledockerin domain and the length of the linker between the dockerin domains is only nine amino acid residues. Therefore, it is reasonable to assume that XynS20E may be a cellulosomal component and that it can bind tightly to the cellulosome.

In general, the rumen fungal carbohydrate esterases had a broader optimal reaction $\mathrm{pH}$ range $(\mathrm{pH} 5.5$ to 9.0$)$ than did the rumen fungal xylanases (pH 5.5 to 7.0 ) (Huang et al. 2005; Cybinski et al. 1999; Blum et al. 1999). In this study, the optimal conditions for the highest xylanase activity of the recombinant XynS20E were observed at a temperature of $49^{\circ} \mathrm{C}$ and a $\mathrm{pH}$ of 5.8 , while those for the highest carbohydrate esterase activity were observed at a temperature of $58^{\circ} \mathrm{C}$ and a $\mathrm{pH}$ of 8.2 (Fig. 3). In addition, the optimal conditions for the highest enzyme activities of XynS20E-CE1 and XynS20E-GH11 were in accordance with XynS20E (results not shown). There is a 55 amino acid region (position 280-334) containing 18 Gly residues between the CE family 1 domain and the dockerin domains of XynS20E. This characteristic feature has previously been identified in linkers separating the different domains of several fibrolytic enzymes (Fanutti et al. 1995; Fillingham et al. 1999). Unlike the usual Ser/Thr/Pro-rich linker found in most other glycosyl hydrolases (Denman et al. 1996), the XynS20E linker sequence is Gly-rich $33 \%$ of the amino acid residues in the linker). Gly-rich linkers are more flexible and are known to retain the capacity of modules to fold independently and to conserve conformational freedom relative to one another, and hence they are often used to separate functional domains of bi- or multifunctional fusion proteins (Lu and Feng 2008). Thus, we suggested that both $\mathrm{CE}$ and GH domains in XynS20E were able to adopt their original conformation and retained their respective optimal reaction temperature and $\mathrm{pH}$.

Previous studies demonstrate that acetylxylan esterases act in synergy with xylanase to increase the release of acetic acid from xylan and hence facilitate the hydrolysis of xylan (Dupont et al. 1996). In this study, the xylanase activity of the recombinant XynS20E was more than twice that of XynS20E-GH11 for birchwood xylan, while the acetylxylan esterase activity of the recombinant XynS20E was about two times that of XynS20E-CE1 for birchwood xylan (Table 5), suggesting that the $\mathrm{CE}$ and $\mathrm{GH}$ domain of XynS20E contribute synergistically to the efficient hydrolysis of xylan. In addition, the recombinant XynS20E and 
XynS20E-CE1 could release acetic acid from birchwood xylan while the recombinant XynS20E-GH11 did not show acetylxylan esterase activity (Table 5), suggesting that the GH domain in XynS20E had no acetylxylan esterase activity.

In conclusion, the cDNA encoding XynS20E was cloned from ruminal fungus $N$. patriciarum and expressed in E. coli. The recombinant XynS20E exhibited acetylxylan esterase and xylanase activities. To our knowledge, this is the first report of a bifunctional xylanolytic enzyme with acetylxylan esterase and xylanase activities from rumen fungus.

Acknowledgments This research was conducted using funds partially provided by grant NSC 98-2313-B-002-033-MY3 from the National Science Council and grant 97AS-2.1.2-AD-U1(3) from the Council of Agriculture, Republic of China.

\section{References}

Akhnazarova S, Kafarov V (1982) Experiment optimization in chemistry and chemical engineering. Mir, Moscow

Aurilia V, Martin JC, McCrae SI, Scott KP, Rincon MT, Flint HJ (2000) Three multidomain esterases from the cellulolytic rumen anaerobe Ruminococcus flavefaciens 17 that carry divergent dockerin sequences. Microbiology 146:1391-1397

Blum DL, Kataeva IA, Li XL, Ljungdahl LG (2000) Feruloyl esterase activity of the Clostridium thermocellum cellulosome can be attributed to previously unknown domains of XynY and XynZ. J Bacteriol 182:1346-1351

Blum DL, Li XL, Chen H, Ljungdahl LG (1999) Characterization of an acetyl xylan esterase from the anaerobic fungus Orpinomyces sp. strain PC-2. Appl Environ Microbiol 65:3990-3995

Cepeljnik T, Rincon MT, Flint HJ, Logar R (2006) Xyn11A, a multidomain multicatalytic enzyme from Pseudobutyrivibrio xylanivorans $\mathrm{Mz}^{\mathrm{T}}$. Folia Microbiol 51:263-267

Collins T, Gerday C, Feller G (2005) Xylanases, xylanase families and extremophilic xylanases. FEMS Microbiol Rev 29:3-23

Cybinski DH, Layton I, Lowry JB, Dalrymaple BP (1999) An acetylxylan esterase and a xylanase expressed from genes cloned from the ruminal fungus Neocallimastix patriciarum act synergistically to degrade acetylated xylans. Appl Microbiol Biotechnol 52:221-225

Dalrymple BP, Cybinski DH, Layton I, McSweeney CS, Xue GP, Swadling YJ, Lowry JB (1997) Three Neocallimastix patriciarum esterases associated with the degradation of complex polysaccharides are members of a new family of hydrolases. Microbiology 143:2605-2614

Ding SJ, Cao J, Zhou R, Zheng F (2007) Molecular cloning, and characterization ofamodularacetyl xylan esterase fromthe edible strawmushroom Volvariella volvacea. FEMS Microbiol Lett 274:304-310

Denman S, Xue GP, Patel B (1996) Characterization of a Neocallimastix patriciarum Cellulase cDNA (celA) Homologous to Trichoderma reesei Cellobiohydrolase II. Appl Environ Microbiol 62:1889-1896

Dupont C, Daigneault N, Shareck F, Morosoli R, Kluepfel D (1996) Purification and characterization of an acetyl xylan esterase produced by Streptomyces lividans. Biochem J 319:881-886

Fanutti C, Ponyi T, Black GW, Hazlewood GP, Gilbert HJ (1995) The conserved noncatalytic 40-residue sequence in cellulases and hemicellulases from anaerobic fungi functions as a protein docking domain. J. Biol. Chem. 270:29314-29322

Ferreira LMA, Wood TM, Williamson G, Faulds C, Hazlewood GP, Black GW, Gilbert HJ (1993) A modular esterase from Pseudomonas fluorescens subsp. cellulosa contains a noncatalytic cellulose-binding domain. Biochem J 294:349-355

Fillingham IJ, Kroon PA, Williamson G, Gilbert HJ, Hazlewood GP (1999) A modular cinnamoyl ester hydrolase from the anaerobic fungus Piromyces equi acts synergistically with xylanase and is part of a multiprotein cellulose-binding cellulase-hemicellulase complex. Biochem J 343:215-224

Halgasova N, Kutejova E, Timko J (1994) Purification and some characteristics of the acetylxylan esterase from Schizophyllum commune. Biochem J 298:751-755

Hall TA (1999) BioEdit: a user-friendly biological sequence alignment editor and analysis. Nucl Acids Symp 41:95-98

Heck JX, Flores SH, Hertz PF, Ayub MAZ (2006) Statistical optimization of thermo-tolerant xylanase activity from Amazon isolated Bacillus circulans on solid-state cultivation. Biores Technol 97:1902-1906

Huang YH, Huang CT, Hseu RS (2005) Effects of dockerin domains on Neocallimastix frontalis xylanases. FEMS Microbiol Lett 243:455-460

Johnson KG, Fontana JD, MacKenzie CR (1988) Measurement of acetylxylan esterase in Streptomyces. Methods Enzymol 160:551560

Khandeparker R, Numan MT (2008) Bifunctional xylanases and their potential use in biotechnology. J Ind Microbiol Biotechnol $35: 635-644$

Konig J, Grasser R, Pikor H, Vogel K (2002) Determination of xylanase, $\beta$-glucanase, and cellulase activity. Anal Bioanal Chem 374:80-87

Kosugi A, Murashima K, Doi RH (2002) Xylanase and acetyl xylan esterase activities of XynA, a key subunit of the Clostridium cellulovorans cellulosome for xylan degradation. Appl Environ Microbiol 68:6399-6402

Laemmli UK (1970) Cleavage of structural proteins during the assembly of the head of bacteriophage T4. Nature 227:680 685

Lineweaver H, Burk D (1934) The determination of enzyme dissociation constants. J Am Chem Soc 57:685

Liu JR, Duan CH, Zhao X, Tzen JTC, Cheng KJ, Pai CK (2008) Cloning of a rumen fungal xylanase gene and purification of the recombinant enzyme via artificial oil bodies. Appl Microbiol Biotechnol 79:225-233

Lu P, Feng MG (2008) Bifunctional enhancement of a $\beta$-glucanasexylanase fusion enzyme by optimization of peptide linkers. Appl Microbiol Biotechnol 79:579-587

Nagy T, Tunnicliffe RB, Higgins LD, Walters C, Gilbert HJ, Williamson MP (2007) Characterization of a double dockerin from the cellulosome of the anaerobic fungus Piromyces equi. J Mol Biol 373:612-622

Raghothama S, Eberhardt RY, Simpson P, Wigelsworth D, White P, Hazlewood GP (2001) Characterization of a cellulosome dockerin domain from the anaerobic fungus Piromyces equi. Nature Struct Biol 8:775-778

Sambrook J, Russell DW (2001) Molecular cloning: a laboratory manual, 3rd edn. Cold Spring Harbor Laboratory Press, Cold Spring Harbor, NY

Segurola J, Allen NS, Edge M, McMahon A (1999) Design of eutectic photoinitiator blends for UV/visible curable acrylated printing inks and coatings. Prog Org Coat 37:23-37

Selinger LB, Forsberg CW, Cheng KJ (1996) The rumen: a unique source of enzymes for enhancing livestock production. Anaerobe 2:263-284 
Shao W, Wiegel J (1995) Purification and characterization of two acetyl xylan esterases from Thermoanaerobacterium sp. strain JW/SL-YS485. Appl Environ Microbiol 1764:263-274

Steenbakkers PJM, Li XL, Ximenes EA, Arts JG, Chen H, Ljungdahl LG, Op Den Camp HJM (2001) Noncatalytic docking domains of cellulosomes of anaerobic fungi. J Bacteriol 183:5325-5333

Subramaniyan S, Prema P (2002) Biotechnology of microbial xylanases: enzymology, molecular biology, and application. Crit Rev Biotechnol 22:33-64
Wakarchuk WW, Campbell RL, Sung WL, Davoodi J, Yaguchi M (1994) Mutational and crystallographic analyses of the active site residues of the Bacillus circulans xylanase. Protein Sci 3:467475

Xie G, Bruce DC, Challacombe JF, Chertkov O, Detter JC, Gilna P, Han CS, Lucas S, Misra M, Myers GL, Richardson P, Tapia R, Thayer N, Thompson LS, Brettin TS, Henrissat B, Wilson DB, McBride MJ (2007) Genome sequence of the cellulolytic gliding bacterium Cytophaga hutchinsonii. Appl Environ Microbiol 73:3536-3546 